\title{
Erectile Dysfunction and Cardiovascular Disease: A Review
}

\begin{abstract}
Erectile dysfunction (ED) is defined as the inability to achieve or maintain a penile erection for satisfactory sexual performance and it is estimated that $>300$ million men will suffer from this condition in 2025 . ED is recognized as a harbinger of cardiovascular disease. Endothelia dysfunction and macrovascular atherosclerotic disease together represent the probable pathophysiological link between vasculogenic $E D$, coronary artery disease (CAD) and peripheral vascular disease (PAD), and research in these common areas in recent years has led to the emergence of a compelling body of evidence to support erectile dysfunction as the sentinel clinical event. The bidirectional relationship between low testosterone and components of metabolic syndrome (MetS) supports the conclusion that normal sex hormone production is an integral component for both metabolic and sexual health for the development of subsequent cardiac events and an improvement in overall health.

Recent findings suggest that hormonal and cardiovascular investigations in men with erectile dysfunction even without any obvious cardiovascular disease (CVD), may ultimately lead to early identification and treatment of cardiovascular risk factors and a reduction in the later development of cardiac events and an improvement in overall health. Such investigation might include assessment of serum testosterone, fasting glucose, $\mathrm{HgbAlc}, \mathrm{C}$ reactive protein and lipid levels as well as selective non-invasive testing.
\end{abstract}

\section{Keywords}

Erectile Dysfunction; Cardiovascular Disease; Endothelial Dysfunction, Risk

\section{Introduction}

Despite its early description and documentation in 1948, erectile dysfunction (ED) has only been recognized as an organic failure of the normal neurovascular function of the penis within the past 2 to 3 decades [1]. Defined as the inability to achieve or maintain a penile erection for satisfactory sexual performance, ED affects $>50 \%$ of men aged 40 to 70 years and $70 \%$ of men aged 70 years or older $[2,3]$. Moreover, the prevalence increases with age and is expected to further rise, potentially affecting over 300 million men worldwide by the year 2025 [4]. Organic, vasculogenic ED can result from aberrations in arterial and venous flow, endothelial and cavernosal smooth muscle function, and tunica albuginea compliance. The most common form of vascular ED results from penile arterial insufficiency, which will serve as the main focus for this review. Atherosclerotic occlusion or narrowing of the common iliac arteries, internal iliac arteries, and the internal pudendal arteries and their downstream branches may cause ED. Chronic arteriolar insufficiency leads to diminished neuronal and endothelial nitrous oxide (NO), and therefore causes impaired cavernosal smooth muscle relaxation resulting in ED.

That ED and cardiovascular disease share many risk factors for atherosclerosis is well established and in many patients, particularly those with early onset ED, sexual dysfunction is a predictor of subsequent cardiovascular disease. While it is clear that endothelial

\section{Journal of}

\section{Andrology \& Gynaecology}

\author{
Charles N Walker ${ }^{1 *}$, Stephanie M Meller ${ }^{2}$, Erik \\ Stilp $^{3}$, and Carlos Mena-Hurtado ${ }^{3}$ \\ ${ }^{1}$ Yale University School of Medicine, Department of Surgery, Section \\ of Urology, USA \\ ${ }^{2}$ Yale University School of Medicine, New Haven, CT, USA \\ ${ }^{3}$ Yale University School of Medicine, Department of Internal \\ Medicine, Section of Cardiovascular Medicine, USA
}

Address for Correspondence

Charles Walker MD, Yale University School of Medicine, Department of Urology, 789 Howard Avenue, FMP 323, New Haven, CT 06519, USA, E-mail: charles.walker@yale.edu

Submission: 26 August 2013

Accepted: 07 October 2013

Published: 10 October 2013

dysfunction is central to this relationship, the artery hypothesis theory proposed by Montorsi et al. in 2005 provides a compelling macrovascular model in support of the concept that ED may be the first manifestation of a single clinical disease spectrum that will ultimately progress to include coronary artery disease (CAD) and peripheral artery disease (PAD) at a later stage [5]. Furthermore, low testosterone or hypogonadism has been associated with erectile dysfunction, metabolic syndrome (MetS), and cardiovascular morbidity, as well as arterial stiffness, a major atherogenic risk factor $[6,7]$.

In this review, we will discuss the evidence for the EDcardiovascular disease link and the clinical implications of cardio metabolic risk reduction.

\section{The Relationship between Erectile Dysfunction and Cardiovascular Disease}

The Massachusetts Male Aging Study established the association between $\mathrm{ED}$ and CAD, demonstrating a $39 \%$ probability of complete $\mathrm{ED}$ in men with heart disease and subsequent studies have shown rates of $\mathrm{ED}$ in patients with CAD as high as $75 \%[2,8,9]$.

\section{Atherosclerosis and Macrovascular Disease}

That ED and CAD are distinct manifestations of the same disease process and are tied by macrovascular atherosclerosis and endothelial dysfunction is a concept which has gained increasing acceptance amongst urologists and cardiologists and is evidenced by common independent predictors and a clear association between both disorders. Several important observations have validated this hypothesis: Not only do patients with ED have high rates of both macrovascular atherosclerosis and hyperlipidemia, but in patients with $\mathrm{ED}$, the presence of $\mathrm{CAD}$ is correlated with obstructive atherosclerotic lesions between the aortic bifurcation and the distal internal pudendal artery (IPA) [10,11].

Support for this association comes from the demonstration that ED precedes the development of symptomatic CAD typically by 2-5 years [12-14]. In 2003, Montorsi et al. showed that of 300 consecutive patients presenting with acute coronary syndromes, $71 \%$ had symptoms of sexual dysfunction prior to the onset of CAD symptoms. ED preceded the onset of CAD by 38 months in this study. 
A similar relationship has been shown in patients with asymptomatic diabetes $[9,15]$. In the Prostate Cancer Prevention Trial, 9,457 men randomized to the placebo arm of the study aged 55 and older were evaluated for the association between both incident and prevalent $\mathrm{ED}$ and subsequent development of cardiovascular events. The study found a hazard ratio of 1.25 for subsequent cardiovascular events in men with incident ED and a hazard ratio of 1.45 ( $\mathrm{p}<0.001)$ for subsequent cardiovascular events in men with either incident or prevalent ED. Incident ED represented a risk for CVD that was of the same magnitude as family history of myocardial infarction, cigarette smoking, and hyperlipidemia [16].

In a retrospective cohort of 1660 patients with ED from the Western Australia Erectile Dysfunction Research Dataset, Chew et al. demonstrated a twofold increased risk of incident $\mathrm{CV}$ events with $76 \%$ of patients having an atherosclerotic event between 10 and 15 years subsequent to the onset of $\mathrm{ED}$. The strength of the association between ED and CVD was greatest in men under 40 and between ages $40-49$, where a 7.5 and a 3.5 fold increased risk of an incident CV event, respectively, was found for men with ED. Likewise, in a meta-analysis of 7 cohort studies involving 45,558 patients, Guo et al. found an adjusted RR of 1.47 for CVD events and 1.41 for MI in men with ED.

Further, the severity of ED is associated with the extent of angiographically proven CAD [17]. Multiple studies have correlated ED severity based on IIEF score with angiographic burden of coronary artery plaque $[18,19]$. In a case control study of 224 men referred for elective angiography, Riedner et al. found a 2.3 fold increased risk of angiographically confirmed CAD in men under age 60 with ED. Severity of ED correlated linearly with the number of coronary vessels involved [20]. It has also been shown that the duration of preceding sexual dysfunction is related to the severity of CAD at presentation [12].

Montorsi et al. has presented a model for the macrovascular association between $\mathrm{ED}$ and $\mathrm{CAD}$ that presents a plausible explanation for why $\mathrm{ED}$ precedes $\mathrm{CAD}$ and other symptomatic vascular disease [5]. The artery size hypothesis asserts that if atherosclerosis progresses at relatively the same pace throughout all major arterial beds, symptoms indicative of disease in smaller arterial branches will manifest first as larger vessels can better tolerate the same extent of plaque buildup (Figure 1). The diameter of the cavernosal artery is $1-2 \mathrm{~mm}$ while that of the proximal left anterior descending artery is 3-4 mm. The smaller luminal diameter of the cavernosal artery would therefore be expected to experience compromise in flow as a result of comparable atherosclerotic plaque burden sooner than coronary and other larger arteries. The low prevalence of occult CAD in ED, the high prevalence of $\mathrm{ED}$ in patients with $\mathrm{CAD}$, and the typical appearance of ED symptoms before those of CAD provide support for the artery size hypothesis $[8,9,12,13,21]$.

\section{Cardiometabolic Risk and ED}

Further evidence for the link between ED and CVD comes from the fact that the conditions share common risk factors including hyperlipidemia, smoking, obesity, and diabetes [22]. Risk factors for CAD have been shown to be present in $92 \%$ of patients with $\mathrm{ED}$ [22]. In one study of 440 men with $\mathrm{ED}, 64 \%$ were smokers, $30 \%$ had diabetes, and $34 \%$ had hyperlipidemia. Further, the number and severity of arterial risk factors is associated with the extent of sexual dysfunction [13]. The National Health and Nutrition Survey

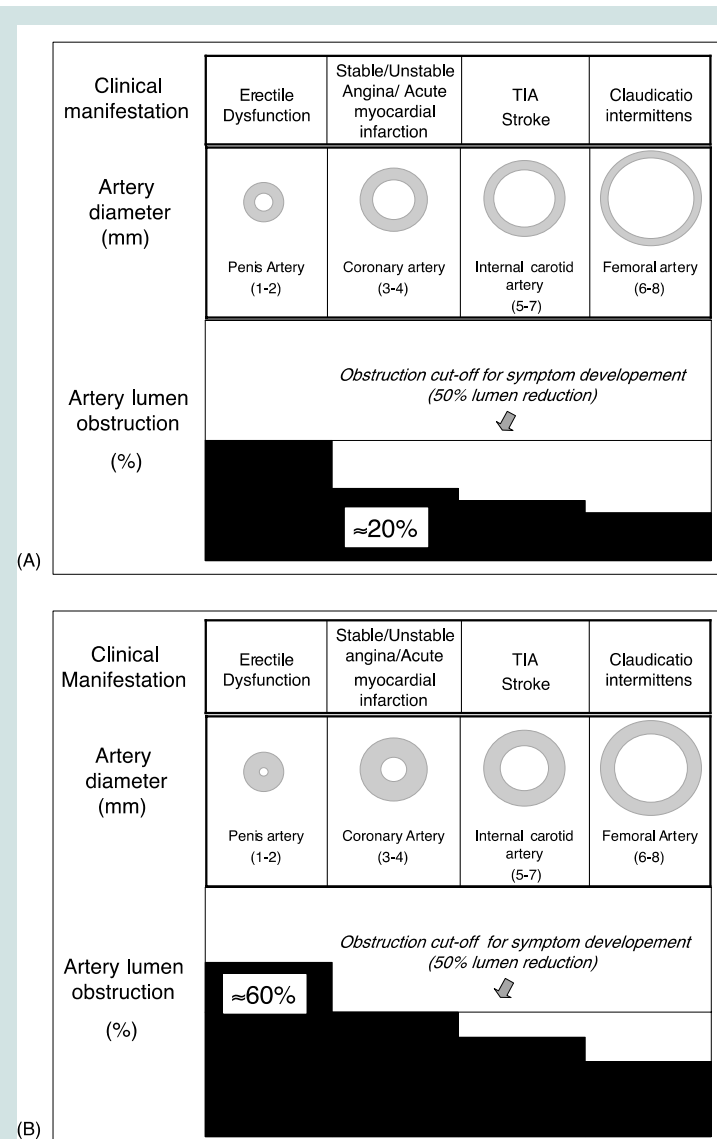

Figure 1: The Artery Size Hypothesis (Montorsi P et al. Is There a Link between Erectile Dysfunction and Coronary Artery Disease? EAU Update Series 2 (2004) 43-48).

(NHANES) demonstrated a prevalence of $77.5 \%$ of ED in men over 75 years of age and DM, obesity, smoking, and hypertension were shown to be independently associated with ED with an OR of 2.69, $1.60,1.74$, and 1.56 respectively [23]. These findings were supported by a prospective study of 132 men with ED, which utilized duplex ultrasound after papaverine injection to assess penile vascular dysfunction in relation to smoking, diabetes, and hypertension. The proportion of abnormal penile vascular findings was associated with the number of cardiometabolic risk factors [24].

\section{Metabolic Syndrome}

ED and MetS are closely linked. In an analysis of 253 men from the Massachusetts Male Aging Study, ED at baseline predicted subsequent MetS in men with a BMI under 25 with a RR of 2.09 [25]. Heidler and colleagues subsequently demonstrated in a crosssectional analysis of a cohort of 2371 men, that the metabolic syndrome and increased waist-to-hip ratio were independently associated with ED as defined by a decrease in the IIEF-5. Men over 50 with MetS were found to have a $48 \%$ increased risk of severe ED [26]. Esposito et al. demonstrated a two-fold increased prevalence of ED ( $26 \%$ vs $13 \%)$ in men with MetS as compared to age and weight matched controls without MetS. Prevalence of ED also increased as the number of components of MetS increased [27].

\section{Hyperlipidemia}

ED is also associated with hyperlipidemia, independent of CAD. 
A large prospective study of 3,250 men found that each $1 \mathrm{mmol} / \mathrm{L}$ increase in total cholesterol and each $1 \mathrm{mmol} / \mathrm{L}$ decrease in highdensity lipoprotein were associated with a 1.32 -fold and 2.6 -fold greater risk of $\mathrm{ED}$, respectively. In men without $\mathrm{CAD}$, circulating soluble oxidized low-density lipoprotein (LDL) receptor levels were also associated with ED in this study [28,29].

\section{Diabetes Mellitus}

The relationship between ED and CVD in men with DM is also well established. In a study of 2,306 diabetic men without clinical evidence of CAD, 27\% had ED. After four years of follow-up, ED was a significant independent predictor of CAD with a HR of 1.58 after adjustment for age, duration of disease, and other covariates [15]. The prevalence of ED is also independently associated with age and duration of DM [30]. In a study of 291 diabetic men with prevalent $\mathrm{ED}$ and angiographically proven "silent" CAD, ED was found to be a powerful predictor of subsequent major adverse cardiac events with a hazard ratio of 2.1 [31].

The occurrence of ED in DM is mediated by insulin resistance, which leads to endothelial dysfunction and atherosclerosis $[25,32,33]$. This has been demonstrated in epidemiological studies and further supported by animal studies demonstrating that insulin administration relieves diabetes-associated ED in rats and increases mean intracavernous pressure following electrostimulation of the cavernous nerves, a surrogate for erectile function [34]. In a rat model, insulin resistance has been associated with lower levels of vascular endothelial growth factor and its receptors, as well as a proapoptotic state in the penile tissue. Also, in a prospective, randomized clinical trial of thirty men without diabetes, the addition of metformin to sildenafil resulted in improved erectile function, suggesting that insulin regulation is involved in the pathogenesis of vasculogenic ED even in the absence of overt diabetes mellitus [35].

\section{Endothelial Dysfunction}

It has been proposed that atherosclerosis causes ED not simply through macrovascular reduction of arterial inflow below an acceptable threshold, but also as a result of inflammation and structural damage to penile cavernosal endothelium and smooth muscle.

Endothelial dysfunction occurs both as a result of impaired NO release and failure of endothelial-dependent vasodilation of vascular smooth muscle. The production of NO by endothelial NO synthase (eNos) in response to shear stress is central to the maintenance of penile erection after initiation by neuronal NO from cavernosal NANC nerve terminals. After release, NO activates guanylate cyclase in the cavernosal smooth muscle cells, increasing the conversion of guanosine triphosphate into cyclic guanosine monophosphate (cGMP). Through a protein kinase cascade, cGMP leads to activation of $\mathrm{Ca}^{2+}$ and $\mathrm{K}^{+}$sensitive channels resulting in hyperpolarization, intracellular calcium sequestration, cavernosal smooth muscle relaxation, arteriolar vasodilation, and erection (Figure 2) [36]. Phosphodiesterase type 5 (PDE-5) mediates return to flaccidity via hydrolysis of cGMP [37].

Early impairment of endothelial dependent vasodilation has been shown in both vasculogenic ED and CAD. Without bioavailable NO, the vascular smooth muscle fails to relax and impedes vasodilation, necessary for erectile function. Kaiser et al. evaluated flow mediated vasodilation of the brachial artery in men with vasculogenic $\mathrm{ED}$, as

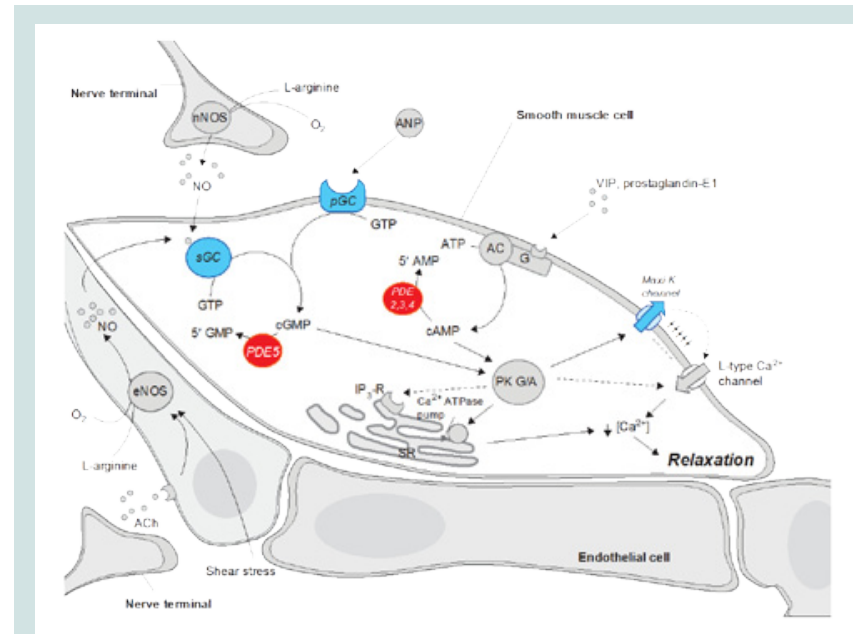

Figure 2: Molecular Physiology of cavernosal smooth muscle relaxation (Albersen M, Shindel AW, Mwamukonda KB, Lue TF: The future is today: emerging drugs for the treatment of erectile dysfunction. Expert Opin Emerg Drugs 2010;15: 467-480).

confirmed by penile Doppler, compared to age matched controls without ED. Men with ED had significantly impaired brachial artery vasodilation comparable to that seen in men with hyperlipidemia and early atherosclerotic disease. Treatment with PDE- 5 inhibitors led to improvement in ED suggesting that the impairment in vasodilation involves the NO-cGMP system. Responsiveness to sublingual nitroglycerin was also reduced suggesting that the defect directly involves smooth muscle. That the impairment of vasodilation was shown to be both endothelium dependent and independent in this study is consistent with both a defect in eNOS bioavailability and intrinsic dysfunction of the smooth muscle. The authors concluded that in addition to the artery size hypothesis, a very plausible explanation for the manifestation of ED before other forms of vascular disease is the dependence of the penile vasculature on NO for vasodilation to a degree much greater than is seen in other vascular beds [38].

In patients with $\mathrm{CAD}$ and $\mathrm{PAD}$, common cardiovascular risk factors create a pro-inflammatory, highly oxidative stressful state that stimulates free radical formation and atherogenesis leading to impaired NO release in such vascular beds. Similarly, obesity, diabetes, and MetS represent chronic inflammatory states and have been shown to damage the vascular endothelium and impair NO release in men with ED [39-41]. Men with obesity have been found to have impairment in multiple indices of endothelial function and significantly elevated levels of C-reactive protein [42]. In other studies recombinant $\mathrm{C}$ - reactive protein has been shown to downregulate endothelial NO and to promote endothelial apoptosis [43]. In a study of overweight patients with ED and without clinical evidence of DM, peripheral vascular disease, or CAD, circulating levels of endothelial progenitor cells (EPC) were reduced as compared to matched controls without $\mathrm{ED}$, the extent of which correlated with severity of ED. Reduced levels of EPC, shown to be an independent predictor of cardiovascular disease, were also shown to independently predict ED in multivariate analysis [44]. Free radical formation, in addition to reducing bioavailability of $\mathrm{NO}$, also potentiates atherosclerosis through direct damage to the endothelium. NO inhibits platelet aggregation and adhesion and smooth muscle proliferation. Thus 
reduced bioavailability of $\mathrm{NO}$ is associated with vasoconstriction, platelet adhesion, and smooth muscle cell proliferation, which further the atherosclerotic burden of the penile vasculature [45].

Further evidence for the role of endothelial dysfunction in ED comes from studies in diabetic men with ED and in animal models. A comparative study on corpus cavernosal and penile resistance arterial tissue from diabetic and non-diabetic men with ED demonstrated that the functional deficiency of NO found in men with ED is exacerbated in diabetes, which correlates with impaired endotheliumdependent relaxation in these patients [46]. Platelet aggregation and blood pressure response to L-arginine administration, surrogates for endothelial function, are believed to be mediated by NO, and have been shown to be lower in diabetic men with ED compared to diabetic patients without ED [47]. In addition, patients with ED have higher levels of asymmetric dimethylarginine, a known inhibitor of eNOS [48]. Insulin-like growth factor binding protein, which regulates the availability of insulin-like growth factor (IGF-1), is increased in hyperglycemic rats. Treatment with IGF-1 results in improvement in rat intracavernosal pressure and expression of endothelial NOsynthase [49-51]. Further evidence for the role of insulin resistance on endothelial NO production comes from the observation in obese rats that metformin administration induces eNos expression in penile tissue, via activated protein kinase [52].

Independent of NO regulation, there is evidence for the role of endothelin-1 in endothelial dysfunction in diabetes. Insulin stimulates production of endothelin-1, a potent vasoconstrictor, and NO in vascular endothelium. In non-diabetic individuals the vasodilatory effects of $\mathrm{NO}$ predominate; however, in insulin resistant states this does not occur. Accordingly, preservation of endothelin in the face of impaired nitric oxide production has been demonstrated in insulin resistant states [33].

\section{Testosterone Deficiency, Metabolic Syndrome, and ED}

The relationship between testosterone deficiency and the MetS is significant and bidirectional [53]. Testosterone deficiency has been shown to be independently associated with the MetS as well as individual components of the syndrome. The two processes are tied by a number of shared pathophysiologic pathways including insulin resistance, hyperglycemia, dyslipidemia, visceral fat accumulation, inflammation, and endothelial dysfunction (Figure 3). Evidence for this comes from studies demonstrating that low levels of total testosterone in patients with $\mathrm{ED}$ have been associated with higher BMI, waist circumference, MetS, and insulin resistance, and treatment with testosterone can reduce central adiposity and insulin resistance $[6,54,55]$. In a prospective study of 1709 men, Kupelian et al. showed that low levels of total testosterone sex hormone binding globulin and symptomatic androgen deficiency were predictive of developing MetS with a RR of 1.41, 1.65, and 2.51 respectively [25] The reciprocal nature of the relationship between the MetS and low testosterone is supported by a study of aging adults with low testosterone the results of which suggest a causative role for MetS in the development of hypogonadism [56]. Components of the MetS may directly promote hypogonadism through a number of mechanisms including decreased production of testosterone by Leydig cells, which is mediated by increased leptin levels in obesity. Visceral obesity and diabetes are both pro-inflammatory states and the production of a number of inflammatory cytokines have been shown to directly inhibit testosterone production, specifically TNF- $\alpha$ [57]. Central obesity leads to increased aromatase activity resulting in conversion

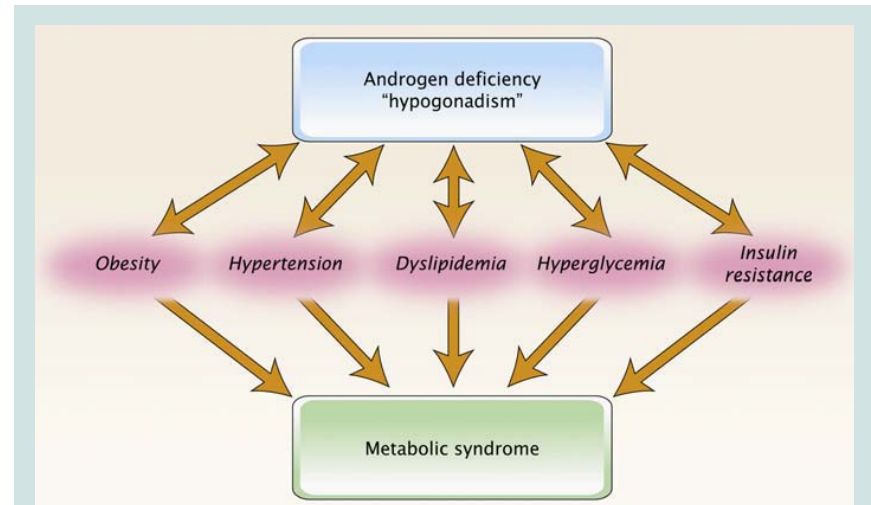

Figure 3: Relationship between hypogonadism and components of the metabolic syndrome (Traish AM, Saad F, Guay A. The dark side of testosterone deficiency: II. Type 2 diabetes and insulin resistance. J Androl 2009; 30:23-32)

of testosterone to increased levels of estradiol (E2), which suppresses LH release through hypothalamic negative feedback resulting in a functional state of hypogonadotropic hypogonadism [58].

The role that testosterone deficiency plays in the evolution of both ED and CAD remains complex and controversial and while a full investigation of this subject is beyond the scope of this review, the topic is worthy of further discussion. It is generally well accepted that libido, frequency of sexual activity, and spontaneous (morning, nocturnal) erections are all testosterone dependent. Support for this comes from studies on hypogonadal men treated with testosterone replacement therapy [59]. Testosterone exerts its effects on erectile function in a number of ways. Centrally, testosterone acts directly on supraspinal centers of sexual function in the preoptic area and paraventricular nucleus of the hypothalamus, where it stimulates the production and release of erectogenic neurotransmitters, such a dopamine and oxytocin. Peripherally, testosterone is required for the normal function of sacral spinal neurons involved in reflexogenic erections and it regulates parasympathetic nerves in corpus cavernosum. Animal studies have shown that testosterone regulates the production of both endothelial and neuronal nitric oxide synthases and androgen suppression has been shown to cause significant reduction in both NOS activity and that of cGMP in the corpus cavernosum $[60,61]$.

Testosterone is also directly required for maintenance of the integrity of both the endothelium and the smooth muscle. Low testosterone is associated with impaired flow mediated vasodilation, a surrogate for endothelial dysfunction [62]. In animal models of castration, atrophy and fibrosis of corporal cavernosal tissue is found to be in part due to the reduction of trabecular smooth muscle content and an increase in components of extracellular matrix [61]. Androgen withdrawal also leads to apoptosis of corporal cavernosal smooth muscle [63]. Animal models and human studies have shown that PDE5 expression is also regulated by testosterone [64,65]. In hypogonadal men with ED that is refractory to PDE5 inhibitors, supplementation with testosterone have been shown to improve erectile function and increase penile arterial flow as indicated by increased cavernosal peak systolic velocities $[66,67]$. Further evidence for the role of testosterone in the maintenance of normal erectile physiology comes from animal studies showing relaxation of corporal smooth muscle after treatment with testosterone [63]. 
Clinically, low levels of total and bioavailable testosterone have been associated with erectile dysfunction [68]. Low testosterone is associated with both hypoactive desire and erectile dysfunction in a dose dependent fashion such that impairment of sexual function is seen when testosterone levels drop below the lower limit of the normal adult range [69]. Corona and colleagues found in their study of 1647 men that low testosterone levels were associated with the severity of ED and the magnitude of penile blood flow [70].

However, reports of the prevalence of hypogonadism in men with $\mathrm{ED}$ are variable in the literature and depend in part on the threshold of testosterone used, whether total testosterone (TT), free testosterone (FT) or bioavailable testosterone (BT) are used to determine prevalence as well as whether or not determinations of testosterone are repeated. Assessment of total testosterone in men with ED reveals a prevalence of hypogonadism between 5-15\% with rates of $20-40 \%$ being found when determinations of free and bioavailable testosterone are used [71-73]. Threshold levels of testosterone for impairment of sexual function are highly variable between individuals. Consequently there is no standardized lower limit of testosterone, however several definitions have been proposed. The lower limit of normal for total testosterone accepted by the FDA is $300 \mathrm{ng} / \mathrm{dL}$ while the recommendation from a 2007 position paper by the Endocrine Society is a testosterone level of $<200 \mathrm{ng} / \mathrm{ml}(6.9$ nM) [74]. For practical purposes the determination of hypogonadism depends on both the determination of testosterone below a threshold level and the presence of symptoms, including reduced sexual drive, impotence, infertility, fatigue, depression or irritability, decreased bone density, loss of lean muscle mass, anemia, and increased body fat.

Both the production and bioavailability of testosterone are reduced with aging at a rate of $0.5 \%-2 \%$ per year [75-78].

\section{ED, Low Testosterone, and Cardiovascular Mortality}

Given the extent of the association between low testosterone, MetS, endothelial dysfunction, ED, and CVD, it is not surprising that both low testosterone and ED are independent predictors of increased cardiovascular mortality [70]. The relationship between ED and mortality has been demonstrated in a number of studies. Vlachopoulos et al. in their meta-analysis of 14 cohort studies found a RR of 1.19 and 1.23 for cardiovascular and all cause mortality respectively in men with $\mathrm{ED}$ [79]. In their meta-analysis of 7 cohort studies of Guo et al. also found that men with ED were at increased risk for all cause mortality with a RR of 1.23 though only two studies reported information on all cause mortality [80]. In a case control study of 291 diabetic men with angiographically confirmed silent CAD, ED significantly predicted an increased risk for major cardiac event defined as CAD Death, sudden death, nonfatal myocardial infarction, death due to congestive heart failure, unstable angina, need for repeat revascularization, stroke or TIA, and symptomatic peripheral artery disease with a hazard ratio of 2.1 [31].

Likewise, the association between low serum testosterone and mortality has been demonstrated in a number of studies. In a retrospective cohort by Shores et al. of 858 male veterans compared all cause mortality rates between men with normal low or equivocal testosterone levels. This study found that even after adjustment for age, medical comorbidity, BMI, glucocorticoid and opiate treatment, race and other covariates men with low testosterone had significantly greater mortality risk with a hazard ratio of 1.88 when compared to men with equivocal and normal testosterone [81]. Though compelling, the study was limited by its retrospective methodology. Further support for this association comes from a prospective population based prospective study of 794 men which found that men with low testosterone were $40 \%$ (HR 1.40) more likely to die over an average follow-up period of 11.8 years than men with normal testosterone. In a separate cause specific analysis low testosterone predicted cardiovascular disease with a hazard ration of 1.38 [82]. Not surprisingly in this study low testosterone was associated with central obesity, HTN, insulin resistance, and lipid profile. In a meta-analysis of 70 studies, Corona et al. evaluated the relationship between hypogonadism and cardiovascular mortality finding that baseline testosterone levels were lower among patients with incident overall and CV mortality. In this study low testosterone was associated with higher estradiol levels, which also correlated with increased risk of CVD and CV mortality [70]. Likewise, in a prospective population based study of 1954 men, low serum testosterone predicted increased all-cause mortality and death from cardiovascular disease after adjustment for covariates with a hazard ratio of 2.32 and 2.56 respectively, which was independent of age, smoking habit, alcohol intake, waist circumference, and physical activity [83]. While these data collectively are not sufficient to establish the causality of hypogonadism in the etiology of CVD they do confirm that a significant association exists. That low testosterone is simply a surrogate for poor overall health or that CV disease causes hypogonadism remain possible explanations. Corona et al. have suggested that hypogonadism could occur as a consequence of CVD and may be an adaptive mechanism hypothesizing that turning off testosterone dependent functions could gave a protective effect in CVD [70].

The close association between low testosterone and elements of the MetS and CVD, suggests that testosterone replacement therapy in hypogonadal men with ED at risk for CVD would provide a means for cardio metabolic risk reduction. Several studies have demonstrated the beneficial effects of TRT on traditional CV risk factors. In a metaanalysis of hypogonadal men, TRT led to an improvement in sexual function and lipid profile as well as reduction in fat mass [84]. In other studies TRT in diabetic patients has been associated with an improvement in insulin resistance [85]. Reductions in total and LDL cholesterol, waist circumference, and triglycerides and improvement in HDL and fasting glucose have been observed in men with MetS and DM on TRT $[54,86]$. Few studies have assessed hypogonadal men for the potential beneficial effects of TRT on CVD; however, Corona et al. demonstrated an improvement in exercise tolerance and time to $1 \mathrm{~mm}$ ST segment depression in hypogonadal men receiving TRT [70]. Current literature lacks evidence supporting the reduction of cardiovascular disease with testosterone replacement.

\section{The Role of Risk Modification}

A Selective phosphodiesterase type 5 (PDE-5) inhibitors (sildenafil, vardenafil, and tadalafil) enhance NO-mediated relaxation of the corpus cavernosum via increased intracavernosal cGMP levels, resulting in erection initiation and maintenance [9]. Given their ease of use and excellent safety profile, PDE- 5 inhibitors are recommended as first line drug therapy; however, these drugs demonstrate lower response rates in older men. The reasons for this age discrepancy include an age-associated decrease in endogenous NO production, which is further diminished by other comorbidities [87]. Of the 
$30-40 \%$ of patients who do not initially respond, counseling and daily low-dose administration may be helpful, and in men with low testosterone, testosterone replacement therapy has been shown to improve response to PDE-5 inhibitor therapy by increasing the bioavailable NO in the cavernous smooth muscle tissue [88].

Furthermore, any condition that disturbs the integrity of the NO-cGMP pathway will diminish the efficacy of PDE-5 inhibitors, which depend on the presence of endogenous NO. Such conditions are the same as those that contribute to CAD and PAD, specifically diabetes, endothelial dysfunction, MetS, and hypercholesterolemia, and may explain why up to $50 \%$ of men have a suboptimal response to pharmacological therapy $[8,11]$

If endothelial dysfunction is the link between ED and CAD then the modification of shared risk factors for endothelial function either through pharmacologic or lifestyle interventions would represent an effective strategy to reduce cardiovascular risk and improve sexual function. Indeed both pharmacologic therapy and lifestyle modification have been demonstrated to have beneficial outcomes which have occurred in parallel to improvements in surrogates for endothelial function.

As discussed above, PDE-5 inhibition is the mainstay of pharmacologic therapy for men with ED. Although, men with diabetes and other metabolic derangements demonstrate a decreased response to PDE-5 inhibitors, a meta analysis of 14 randomized controlled trials found a $63 \%$ improvement in erectile function in diabetic men taking sildenafil compared to $19 \%$ of those taking placebo. Men with ischemic heart disease in this study had a $42 \%$ response to sildenafil compared to $14 \%$ of those in the placebo group [89]. It has been proposed that the beneficial effect of PDE- 5 inhibitors on NO production and endothelial function in men with ED may reduce cardiovascular risk in this population. In a randomized placebo controlled study of 32 men with ED and increased cardiovascular risk treated with chronic Tadalafil, Rosano et al. demonstrated an improvement in endothelial function as evidenced by improvement in brachial artery flow mediated vasodilation [90].

In a study of 291 diabetic patients with erectile dysfunction, PDE5 inhibitor and statin use were associated with decreased risk of major adverse cardiac events with a hazard ratio of 0.3 and 0.2 respectively [32]. While no difference in erectile function was associated with statin use in this study, the effect of statins on erectile dysfunction remains unclear. In another study, the addition of $40 \mathrm{mg}$ of atorvastatin to sildenafil in men with ED refractory to PDE 5 inhibitors led to a significant improvement in SHIM score compared to placebo [91]. The administration of statins in combination with a diet low in fat and cholesterol has also been shown to significantly improve erectile function $[92,93]$.

The beneficial effect of sildenafil as a treatment for pulmonary hypertension is well established and has spurred interest in identifying similar benefit for other related vascular diseases [94]. There is evidence from animal studies that PDE5 inhbitors have a protective effect in cardiac ischemia-reperfusion injury [95]. Sildenafil has been shown to lower systemic blood pressure and it has been hypothesized that PDE5 inhibitors may help improve ventricular function in CHF by enhancing flow mediated vasodilation [96,97]. In another study sildenafil treatment led to dilation of epicardial coronary arteries and inhibited platelet activation in patients with CAD [98]. PDE-5 inhibitors are thought to exert their cardio protective effects in part by upregulating eNOS and increasing NO in cardiac myocytes [99].

A number of studies have assessed the effect of lifestyle modification on sexual function and cardiovascular risk. A meta-analysis of 740 patients from 6 clinical trials in 4 countries found that cardiac risk factor treatment (i.e. lifestyle modifications and pharmacotherapy) was associated with significant improvements in IIEF-5 scores and sexual function [100]. In a randomized controlled trial of obese men with erectile dysfunction randomized to receive either an intervention of detailed instruction and counseling on dietary intake, weight reduction, and exercise or general information about healthy eating and exercise, men randomized to the intervention group demonstrated a $31 \%$ improvement in erectile dysfunction [101]. In addition men in the intervention group experienced significant decreases in traditional cardiovascular risk factors including weight, BMI, waist to hip ratio, glucose, insulin, total cholesterol and triglycerides and a significant increase in high density lipoprotein. In an analysis of 1709 men representing a subset of men between the age of 40 and 70 years of age in the MMAS Derby et al. demonstrated that the lowest risk of ED was observed in men who were sedentary at the outset of the study and increased their exercise level over the course of the 8.8 year study follow-up [102]. Interestingly this study also found that neither weight loss nor cessation of smoking were associated with decreased risk of ED. This inverse relationship between baseline level of exercise and ED has also been demonstrated in a number of large cross-sectional population studies $[103,104]$.

Similarly, physical exercise is inversely related to the incidence of cardiovascular disease both in men and in women. Exercise likely reduces cardiovascular risk by increasing coronary blood flow leading to an increase in shear stress, which in turn promotes an increase in endothelial NO production [105]. Accordingly, increases in NO due to exercise have been demonstrated both in dogs and in humans [106]. It follows that in men with ED improvements in endothelial function as a result of exercise will similarly lead to improvement in erectile function.

The role that dietary habits have in the etiology of cardiovascular disease is well established as are the potential benefits of dietary modification in reducing the burden of CVD [107]. It is therefore logical that diet represents a potentially modifiable risk factor for both $\mathrm{ED}$ and $\mathrm{CV}$ health, which share the same pathophysiology of endothelial dysfunction and atherosclerosis. A number of studies have demonstrated the association between adherence to Mediterranean Diet and reduction in risk of overall and cardiovascular mortality $[108,109]$. Similarly, a Mediterranean diet or diets rich in fruits, nuts, vegetables, and fish and low in red meat have been shown to be associated with lower rates of ED [110,111].

\section{Guidelines for Management of the Patient with ED} and No Evidence of Cardiovascular Disease

The accumulation of evidence supporting ED as a predictor of CAD indicates that ED and hypogonadism should serve as potential warning signs of future cardiovascular disease [32]. As proposed by Miner et al., a "window of curability" may exist in which treatment may stop the progression of cardiovascular disease and potentially $\mathrm{ED}$, especially in men $<60$ years old, where the cardiovascular risk portended by ED appears to be especially high $[53,112]$.

The Princeton III consensus, an expert panel convened in 2010 to specifically address the relationship between sexual function and 
cardiovascular health, endorses the use of Framingham Risk Score (FRS) as well as the American College of Cardiology Foundation/ American Heart Association (ACCF/AHA) guidelines for the assessment of CV risk in men presenting with ED [113]. However the panel also concluded that ED alone is a marker for increased risk of CV disease, independent of the FRS [114,115].

The Princeton III panel recommended that risk assessment for men with ED with and without CVD should include 1) a thorough history emphasizing identification of comorbid conditions, family history, lifestyle factors 2) physical examination with attention to waist circumference, BMI, peripheral pulses, and cardiac auscultation 3) assessment of ED severity and duration 4) resting EKG, 5) fasting plasma glucose 6) serum creatinine and albumin to creatinine ratio and 7) Total testosterone and 8) plasma lipid levels [113].

The panel recommends that all men with $\mathrm{ED}>30$ years of age undergo non-invasive evaluation, specifically exercise stress testing as well as CT for coronary artery calcium scoring, carotid intima media thickness, pulse wave velocity, ankle brachial index, and brachial artery flow-mediated dilation. Use of non-traditional investigations for $\mathrm{CV}$ risk should be considered and may include: c-reactive protein, hemoglobin A1c, and urinary albumin excretion. Lipoproteinassociated phospholipase A2 levelsmay be useful in delineating risk of subclinical CVD in intermediate risk patients $[114,116]$.

\section{Conclusion}

The link between vasculogenic ED and CVD is well supported by the literature and very likely indicates that the disease processes are distinct manifestations of the same atherosclerotic process. ED may be the first overt manifestation of potentially serious cardiovascular disease. Because vasculogenic ED typically presents earlier than CVD, clinicians should recognize the condition as an early warning sign of future cardiovascular pathology particularly in diabetic men.

As recommended in the Princeton III guidelines, all patients with presumed vasculogenic ED warrant assessment of cardiovascular risk factors, screening for occult cardiovascular disease, and aggressive modification of atherosclerotic risk factors, especially those with a suboptimal response to PDE-5 inhibition. A reasonable approach to risk modification in this population would be one aimed at reducing inflammation and promoting endothelial health. This will necessitate not only pharmacological interventions aimed at controlling CV risk factors like hyperlipidemia and hypertension but also incorporation of lifestyle interventions focusing on smoking cessation, exercise, weight loss, and the adoption of diets low in red meat and rich in fruits, vegetables, fish, and nuts. all men presenting with ED should undergo baseline testosterone assessment. Testosterone replacement therapy should be considered for symptomatic patient with low testosterone and may improve cardiovascular risk factors in patients with MetS. As we look toward the future given the inevitability of associated cardiovascular risk factor in men with ED, the management of ED will increasingly require a multidisciplinary approach in which communication and collaboration between urologists, primary care and family practioners, cardiologists, endocrinologists, and health psychologists will be paramount.

\section{References}

1. Kinsey AC, Pomeroy WB, Martin CE (1948) Sexual behavior in the human male.
2. Johannes $C B$ AA, Feldman HA, Derby CA, Kleinman KP, McKinlay JB et al. (2000) Incidence of erectile dysfunction in men 40 to 69 years old: Longitudinal results from the massachusetts male aging study. J Urol 163: 460-463.

3. Chew KK, Finn J, Stuckey B, Gibson N, Sanfilippo F, et al. (2010) Erectile dysfunction as a predictor for subsequent atherosclerotic cardiovascular events: Findings from a linked-data study. J Sex Med 7: 192-202.

4. Aytac IA, Araujo AB, Johannes CB, Kleinman KP, McKinlay JB (2000) Socioeconomic factors and incidence of erectile dysfunction: Findings of the longitudinal massachussetts male aging study. Soc Sci Med 51: 771-778.

5. Montorsi P, Ravagnani PM, Galli S, Rotatori F, Briganti A, et al. (2005) The artery size hypothesis: A macrovascular link between erectile dysfunction and coronary artery disease. Am J Cardiol 96: 19M-23M.

6. Guay A, Jacobson J (2007) The relationship between testosterone levels, the metabolic syndrome (by two criteria), and insulin resistance in a population of men with organic erectile dysfunction. J Sex Med 4:1046-1055.

7. Hougaku H, Fleg JL, Najjar SS, Lakatta EG, Harman SM, et al. (2006) Relationship between androgenic hormones and arterial stiffness, based on longitudinal hormone measurements. Am J Physiol Endocrinol Metab 290: E234-242.

8. Kloner RA, Mullin SH, Shook T, Matthews R, Mayeda G, et al. (2003) Erectile dysfunction in the cardiac patient: How common and should we treat? J Urol 170: S46-50.

9. Montorsi F, Briganti A, Salonia A, Rigatti P, Margonato A, et al. (2003) Erectile dysfunction prevalence, time of onset and association with risk factors in 300 consecutive patients with acute chest pain and angiographically documented coronary artery disease. Eur Urol 44: 360-364

10. Kaiser FE, Viosca SP, Morley JE, Mooradian AD, Davis SS, et al. (1988) Impotence and aging: Clinical and hormonal factors. J Am Geriatr Soc 36: 511-519.

11. Rogers JH, Karimi H, Kao J, Link D, Javidan J, et al. (2010) Internal pudenda artery stenoses and erectile dysfunction: Correlation with angiographic coronary artery disease. Catheter Cardiovasc Interv 76: 882-887.

12. Montorsi P, Ravagnani PM, Galli S, Rotatori F, Veglia1F, et al. (2006) Association between erectile dysfunction and coronary artery disease. Role of coronary clinical presentation and extent of coronary vessels involvement: The cobra trial. Eur Heart J 27: 2632-2639.

13. Virag R, Bouilly P, Frydman D (1985) Is impotence an arterial disorder? A study of arterial risk factors in 440 impotent men. Lancet 1: 181-184.

14. Montorsi P, Ravagnani PM, Galli S, Briganti A, Salonia A, et al. (2005) Association between erectile dysfunction and coronary artery disease: A case report study. J Sex Med 2: 575-582.

15. Ma RC, So WY, Yang X, Yu LW, Kong AP, et al. (2008) Erectile dysfunction predicts coronary heart disease in type 2 diabetes. J Am Coll Cardiol 51: 2045-2050.

16. Thompson IM TC, Tangen CM, Goodman PJ, Probstfield JL, Moinpour CM, et al. (2005) Erectile dysfunction and subsequent cardiovascular disease. JAMA 294: 2996-3002.

17. Greenstein A CJ, Chen J, Miller H, Matzkin $H$, Villa $Y$, et al (1997) Does severity of ischemic coronary disease correlate with erectile function? Int J Impot Res 9: 123-126.

18. Solomon H, Man JW, Wierzbicki AS, Jackson G (2003) Relation of erectile dysfunction to angiographic coronary artery disease. Am J Cardiol 91: 230231.

19. Foroutan SK, Rajabi M (2007) Erectile dysfunction in men with angiographically documented coronary artery disease. Urol J 4: 28-32.

20. Riedner CE, Rhoden EL, Fuchs SC, Wainstein MV, Gonçalves SC, et al. (2011) Erectile dysfunction and coronary artery disease: An association of higher risk in younger men. J Sex Med 8: 1445-1453.

21. Kawanishi Y, Lee KS, Kimura K, Koizumi T, Nakatsuji H, et al. (2001) Screening of ischemic heart disease with cavernous artery blood flow in erectile dysfunctional patients. Int J Impot Res 13:100-103.

22. El-Sakka Al, Morsy AM, Fagih BI, Nassar AH (2004) Coronary artery risk factors in patients with erectile dysfunction. J Urol 172: 251-254. 
23. Saigal CS, Wessells H, Pace J, Schonlau M, Wilt TJ, et al. (2006) Predictors and prevalence of erectile dysfunction in a racially diverse population. Arch Intern Med 166: 207-212.

24. Shabsigh R, Fishman IJ, Schum C, Dunn JK (1991) Cigarette smoking and other vascular risk factors in vasculogenic impotence. Urology 38: 227-231.

25. Kupelian V, Shabsigh R, Araujo AB, O'Donnell AB, McKinlay JB (2006) Erectile dysfunction as a predictor of the metabolic syndrome in aging men: Results from the massachusetts male aging study. J Urol 176: 222-226.

26. Heidler S, Temml C, Broessner C, Mock K, Rauchenwald M, et al. (2007) Is the metabolic syndrome an independent risk factor for erectile dysfunction? J Urol 177: 651-654.

27. Esposito K, Giugliano F, Martedi E, Feola G, Marfella R, et al. (2005) High proportions of erectile dysfunction in men with the metabolic syndrome. Diabetes care 28: 1201-1203

28. Wei M MC, Davis DR, Hornung CA, Nankin HR, Blair SN, et al. (1994) Tota cholesterol and high density lipoprotein cholesterol as important predictors of erectile dysfunction. Am J Epidemiol 140: 930-937.

29. Kobat MA, Fırdolas F, Balin M, Celik A, Bentli R, et al. (2012) Circulating soluble lectin-like oxidized low-density lipoprotein receptor-1 levels are associated with erectile dysfunction in patients without known coronary artery disease. J Sex Med.

30. Garcia-Malpartida K, Marmol R, Jover A, Gomez-Martinez MJ, SolaIzquierdo E, et al. (2011) Relationship between erectile dysfunction and silen myocardial ischemia in type 2 diabetic patients with no known macrovascular complications. J Sex Med 8: 2606-2616.

31. Gazzaruso C, Solerte SB, Pujia A, Coppola A, Vezzoli M, et al. (2008) Erectile dysfunction as a predictor of cardiovascular events and death in diabetic patients with angiographically proven asymptomatic coronary artery disease: A potential protective role for statins and 5-phosphodiesterase inhibitors. $J$ Am Coll Cardiol 51: 2040-2044.

32. Corona G, Mannucci E, Forti G, Maggi M (2009) Hypogonadism, ed, metabolic syndrome and obesity: A pathological link supporting cardiovascular diseases. Int J Androl 32: 587-598.

33. Stehouwer CD, Henry RM, Ferreira I (2008) Arterial stiffness in diabetes and the metabolic syndrome: A pathway to cardiovascular disease. Diabetologia 51: 527-539

34. Yamanaka M, Shirai M, Shiina H, Tanaka Y, Tsujimura A, et al. (2003) Diabetes induced erectile dysfunction and apoptosis in penile crura are recovered by insulin treatment in rats. J Urol 170: 291-297.

35. Rey-Valzacchi GJ, Costanzo PR, Finger LA, Layus AO, Gueglio GM, et al (2012) Addition of metformin to sildenafil treatment for erectile dysfunction in eugonadal nondiabetic men with insulin resistance. A prospective, randomized, double-blind pilot study. J Androl 33: 608-614.

36. Lue TF (2003) Erectile dysfunction. N Engl J Med 342:1802-1813.

37. Simonsen U, Garcia-Sacristan A, Prieto D (2002) Penile arteries and erection. J Vasc Res 39:283-303.

38. Kaiser DR, Billups K, Mason C, Wetterling R, Lundberg JL,et al. (2004) Impaired brachial artery endothelium-dependent and -independent vasodilation in men with erectile dysfunction and no other clinical cardiovascular disease. Journal of the American College of Cardiology. 43:179-184.

39. Martinez-Jabaloyas JM (2013) Prevalence of co-morbidities in patients with erectile dysfunction. Actas Urol Esp 37: 33-39.

40. Bansal TC GA, Jacobson J, Woods BO, Nesto RW (2005) Incidence of metabolic syndrome and insulin resistance in a population with organic erectile dysfunction. J Sex Med 2:96-103.

41. Potenza MA, Montagnani M (2008) Abnormal insulin signaling: Early detection of silent coronary artery disease-erectile dysfunction? Curr Pharm Des 14: 3737-3748.

42. Giugliano F, Esposito K, Di Palo C, Ciotola M, Giugliano G (2004) Erectile dysfunction associates with endothelial dysfunction and raised proinflammatory cytokine levels in obese men. J Endocrinol Invest 27: 665669.

43. Verma S, Wang CH, Li SH, Dumont AS, Fedak PW, et al. (2002) A selffulfilling prophecy: C-reactive protein attenuates nitric oxide production and inhibits angiogenesis. Circulation 106: 913-919.

44. Esposito K, Ciotola M, Maiorino MI, Giugliano F, Autorino R, et al. (2009) Circulating cd34+ kdr+ endothelial progenitor cells correlate with erectile function and endothelial function in overweight men. J Sex Med 6: 107-114.

45. Creager MA, Luscher TF, Cosentino F, Beckman JA (2003) Diabetes and vascular disease: Pathophysiology, clinical consequences, and medical therapy: Part i. Circulation 108:1527-1532.

46. Angulo J, Gonzalez-Corrochano R, Cuevas P, Fernandez A, La Fuente JM, et al. (2010) Diabetes exacerbates the functional deficiency of no/cgmp pathway associated with erectile dysfunction in human corpus cavernosum and penile arteries. J Sex Med 7: 758-768.

47. De Angelis L, Marfella MA, Siniscalchi M, Marino L, Nappo F, et al. (2001) Erectile and endothelial dysfunction in type ii diabetes: A possible link. Diabetologia 44:1155-1160.

48. Elesber AA, Solomon H, Lennon RJ, Mathew V, Prasad A, et al. (2006) Coronary endothelial dysfunction is associated with erectile dysfunction and elevated asymmetric dimethylarginine in patients with early atherosclerosis. Eur Heart J 27: 824-831.

49. Soh J, Katsuyama M, Ushijima S, Mizutani Y, Kawauchi A, et al. (2007) Localization of increased insulin-like growth factor binding protein-3 in diabetic rat penis: Implications for erectile dysfunction. Urology 70:10191023.

50. Pu XY HL, Wang HP, Luo $Y X$, Wang $X H$ (2007) Improvement in erectile dysfunction after insulin-like growth factor-1 gene therapy in diabetic rats. Asian J Androl 9: 83-91.

51. Pu XY, Wen AM, Zheng XG, Liu JM, Zhou XX, et al. (2012) [insulin-like growth factor-1 gene therapy improves the levels of mrna and protein of endothelial nitric oxide synthase in aging related erectile dysfunction in rats]. Zhonghua yi xue za zhi 92:128-130.

52. Kim YW, Park SY, Kim JY, Huh JY, Jeon WS, et al. (2007) Metformin restores the penile expression of nitric oxide synthase in high-fat-fed obese rats. J Androl 28: 555-560

53. Miner MM (2012) Men's health in primary care: An emerging paradigm of sexual function and cardiometabolic risk. Urol Clin North Am 39:1-23.

54. Knoblovits P, Costanzo PR, Valzacchi GJ, Gueglio G, Layus AO, et al. (2010) Erectile dysfunction, obesity, insulin resistance, and their relationship with testosterone levels in eugonadal patients in an andrology clinic setting. J Androl 31: 263-270.

55. Stanworth RD, Jones TH (2008) Testosterone for the aging male; current evidence and recommended practice. Clin Interv Aging 3: 25-44.

56. Corona G, Monami M, Rastrelli G, Aversa A, Tishova Y, et al. (2011) Testosterone and metabolic syndrome: A meta-analysis study. J Sex Med 8: 272-283.

57. Hong CY, Park JH, Ahn RS, Im SY, Choi HS, et al. (2004) Molecular mechanism of suppression of testicular steroidogenesis by proinflammatory cytokine tumor necrosis factor alpha. Mol Cell Biol 24: 2593-2604.

58. Vermeulen A, Kaufman JM, Deslypere JP, Thomas G (1993) Attenuated luteinizing hormone (lh) pulse amplitude but normal Ih pulse frequency, and its relation to plasma androgens in hypogonadism of obese men. J Clin Endocrinol Metab 76: 1140-1146.

59. Buvat J, Shabsigh R, Guay A, Gooren L, Torres L, Mueleman E. Hormones, metabolism, aging and men's health. In: Porst H, Buvat J, Medicine TSCotISfS, eds.(2006) Standard practice in sexual medicine. Massachusettes, USA: Blackwell Publishing 225-286.

60. Park KH, Kim SW, Kim KD, Paick JS (1999) Effects of androgens on the expression of nitric oxide synthase mRNAs in rat corpus cavernosum. BJU Int 1999 83: 327-333.

61. Traish A, Kim N (2005) The physiological role of androgens in penile erection: Regulation of corpus cavernosum structure and function. J Sex Med 2:759770

62. Akishita M, Hashimoto M, Ohike Y, Ogawa S, lijima K, et al. (2007) Low testosterone level is an independent determinant of endothelial dysfunction in men. Hypertens Res 30:1029-1034.

63. Alcorn JF, Toepfer JR, Leipheimer RE (1999) The effects of castration on 
relaxation of rat corpus cavernosum smooth muscle in vitro. J Urol 161: 686689.

64. Zhang XH, Morelli A, Luconi M, Vignozzi L, Filippi S, et al. (2005) Testosterone regulates pde5 expression and in vivo responsiveness to tadalafil in rat corpus cavernosum. Eur Urol 47: 409-416.

65. Morelli A, Filippi S, Mancina R, Luconi M, Vignozzi L, et al. (2004) Androgens regulate phosphodiesterase type 5 expression and functional activity in corpora cavernosa. Endocrinology 145: 2253-2263.

66. Aversa A, Isidori AM, Spera G, Lenzi A, Fabbri A (2003) Androgens improve cavernous vasodilation and response to sildenafil in patients with erectile dysfunction. Clin Endocrinol 58:632-638.

67. Shabsigh R, Kaufman JM, Steidle C, Padma-Nathan H (2004) Randomized study of testosterone gel as adjunctive therapy to sildenafil in hypogonadal men with erectile dysfunction who do not respond to sildenafil alone. J Urol 172: 658-663.

68. Kratzik CW, Schatzl G, Lunglmayr G, Rucklinger E, Huber J (2005) The impact of age, body mass index and testosterone on erectile dysfunction. J Urol 174: 240-243.

69. Bhasin S, Woodhouse L, Casaburi R, Singh AB, Bhasin D, et al. (2001) Testosterone dose-response relationships in healthy young men. Am J Physiol Endocrinol Metab 281: E1172-1181.

70. Corona G, Rastrelli G, Monami M, Guay A, Buvat J, et al. (2011) Hypogonadism as a risk factor for cardiovascular mortality in men: A metaanalytic study. Eur J Endocrinol 165: 687-701.

71. Buvat J, Lemaire A (1997) Endocrine screening in 1,022 men with erectile dysfunction: Clinical significance and cost-effective strategy. J urol 158:17641767

72. Bodie J, Lewis J, Schow D, Monga M (2003) Laboratory evaluations of erectile dysfunction: An evidence based approach. J urol 169: 2262-2264.

73. Earle CM, Stuckey BG (2003) Biochemical screening in the assessment of erectile dysfunction: What tests decide future therapy? Urology 62: 727-731.

74. Rosner W, Auchus RJ, Azziz R, Sluss PM, Raff H (2007) Position statement: Utility, limitations, and pitfalls in measuring testosterone: An endocrine society position statement. J Clin Endocrinol Metab 2007 92: 405-413.

75. Morley JE, Kaiser FE, Perry HM 3rd, Patrick P, Morley PM, et al. (1997) Longitudinal changes in testosterone, luteinizing hormone, and folliclestimulating hormone in healthy older men. Metabolism 46: 410-413.

76. Mulligan T, Frick MF, Zuraw QC, Stemhagen A, McWhirter C (2006) Prevalence of hypogonadism in males aged at least 45 years: The him study. Int J Clin Pract 60: 762-769.

77. Snyder PJ (2001) Effects of age on testicular function and consequences of testosterone treatment. J Clin Endocrinol Metab 86: 2369-2372.

78. Vermeulen A (2001) Androgen replacement therapy in the aging male--a critical evaluation. J Clin Endocrinol Metab 86:2380-2390.

79. Vlachopoulos CV, Terentes-Printzios DG, loakeimidis NK, Aznaouridis KA Stefanadis $\mathrm{Cl}$ (2013) Prediction of cardiovascular events and all-cause mortality with erectile dysfunction: A systematic review and meta-analysis of cohort studies. Circ Cardiovasc Qual Outcomes 6: 99-109.

80. Guo W, Liao C, Zou Y, Li F, Li T, et al. (2010) Erectile dysfunction and risk of clinical cardiovascular events: A meta-analysis of seven cohort studies. J Sex Med 7: 2805-2816.

81. Shores MM, Matsumoto AM, Sloan KL, Kivlahan DR (2006) Low serum testosterone and mortality in male veterans. Arch Intern Med 166: 1660-1665.

82. Laughlin GA, Barrett-Connor E, Bergstrom J (2008) Low serum testosterone and mortality in older men. J Clin Endocrinol Metab 93:68-75.

83. Haring R, Volzke H, Steveling A, Krebs A, Felix SB, et al. (2010) Low serum testosterone levels are associated with increased risk of mortality in a population-based cohort of men aged 20-79. Eur Heart J 31:1494-1501.

84. Isidori AM, Giannetta E, Greco EA, Gianfrilli D, Bonifacio V, et al. (2005) Effects of testosterone on body composition, bone metabolism and serum lipid profile in middle-aged men: A meta-analysis. Clin Endocrinol 63: $280-$ 293.

85. Boyanov MA, Boneva Z, Christov VG (2003)Testosterone supplementation in men with type 2 diabetes, visceral obesity and partial androgen deficiency. Aging Male 6:1-7.

86. Whitsel EA, Boyko EJ, Matsumoto AM, Anawalt BD, Siscovick DS (2001) Intramuscular testosterone esters and plasma lipids in hypogonadal men: A meta-analysis. Am J Med 111:261-269.

87. Albersen M, Orabi H, Lue TF (2012) Evaluation and treatment of erectile dysfunction in the aging male: A mini-review. Gerontology 58: 3-14.

88. Shabsigh R, Kaufman JM, Steidle C, Padma-Nathan H (2004) Randomized study of testosterone gel as adjunctive therapy to sildenafil in hypogonadal men with erectile dysfunction who do not respond to sildenafil alone. J Urol 172: 658-663.

89. Fink HA, Mac Donald R, Rutks IR, Nelson DB, Wilt TJ (2002) Sildenafil for male erectile dysfunction: A systematic review and meta-analysis. Arch Intern Med 162:1349-1360.

90. Rosano GM, Aversa A, Vitale C, Fabbri A, Fini M, et al. (2005)Chronic treatment with tadalafil improves endothelial function in men with increased cardiovascular risk. Eur Urol 47: 214-220.

91. Herrmann HC, Levine LA, Macaluso J, Jr., Walsh M, Bradbury D, et al. (2006) Can atorvastatin improve the response to sildenafil in men with erectile dysfunction not initially responsive to sildenafil? Hypothesis and pilot trial results. J Sex Med 3: 303-308.

92. Juenemann KP, Muth S, Rohr G, Siegsmund M, Alken P (1990) Does lipid metabolism influence the pathogenesis of vascular impotence? Int $\mathrm{J}$ Impot Res 2: 33.

93. Manning M, Schmidt $P$, Juenemann KP, Alken $P$ ( 1996) The role of blood lipids in erectile failure. Int J Impot Res 8: D179.

94. Ghofrani HA, Pepke-Zaba J, Barbera JA, Channick R, Keogh AM,et al. (2004) Nitric oxide pathway and phosphodiesterase inhibitors in pulmonary arterial hypertension. J Am Coll Cardiol 43: 68S-72S.

95. Salloum FN, Ockaili RA, Wittkamp M, Marwaha VR, Kukreja RC (2006) Vardenafil: A novel type 5 phosphodiesterase inhibitor reduces myocardial infarct size following ischemia/reperfusion injury via opening of mitochondrial k(atp) channels in rabbits. J Mol Cell Cardiol 40: 405-411.

96. Oliver JJ, Melville VP, Webb DJ (2006) Effect of regular phosphodiesterase type 5 inhibition in hypertension. Hypertension 48: 622-627.

97. Katz SD, Balidemaj K, Homma S, Wu H, Wang J, et al. (2000) Acute type 5 phosphodiesterase inhibition with sildenafil enhances flow-mediated vasodilation in patients with chronic heart failure. J Am Coll Cardiol 36: 845851.

98. Halcox JP, Nour KR, Zalos G, Mincemoyer RA, Waclawiw M,et al. (2002) The effect of sildenafil on human vascular function, platelet activation, and myocardial ischemia. J Am Coll Cardiol 40: 1232-1240.

99. Salloum F, Yin C, Xi L, Kukreja RC (2003) Sildenafil induces delayed preconditioning through inducible nitric oxide synthase-dependent pathway in mouse heart. Cir Res 92: 595-597.

100. Gupta BP MM, Clifton MM, Prokop L, Nehra A, et al. (2011) The effect of lifestyle modification and cardiovascular risk factor reduction on erectile dysfunction: A systematic review and meta-analysis. Arch Intern Med 171: 1797-1803.

101. Esposito K, Giugliano F, Di Palo C, Giugliano G, Marfella R, et al. (2004) Effect of lifestyle changes on erectile dysfunction in obese men: A randomized controlled trial. JAMA 291: 2978-2984.

102. Derby CA, Mohr BA, Goldstein I, Feldman HA, Johannes CB, et al. (2000) Modifiable risk factors and erectile dysfunction: Can lifestyle changes modify risk? Urology 56: 302-306.

103. Bacon CG, Mittleman MA, Kawachi I, Giovannucci E, Glasser DB, et al (2003) Sexual function in men older than 50 years of age: Results from the health professionals follow-up study. Ann Intern Med 139:161-168.

104. Nicolosi A, Glasser DB, Moreira ED, Villa M (2003) Prevalence of erectile dysfunction and associated factors among men without concomitant diseases: A population study. Int J Impot Res 15: 253-257. 
105. Niebauer J, Cooke JP (1996) Cardiovascular effects of exercise: Role of endothelial shear stress. J Am Coll Cardiol 28:1652-1660.

106. Hambrecht R, Wolf A, Gielen S, Linke A, Hofer J, et al. (2000) Effect of exercise on coronary endothelial function in patients with coronary artery disease. N Engl J Med 342: 454-460.

107. Lichtenstein AH, Appel LJ, Brands M, Carnethon M, Daniels S, et al. (2006) Diet and lifestyle recommendations revision 2006: A scientific statement from the american heart association nutrition committee. Circulation 114 82-96.

108. Sofi F, Cesari F, Abbate R, Gensini GF, Casini A (2008) Adherence to mediterranean diet and health status: Meta-analysis. BMJ 337: a1344.

109. Fung TT, Rexrode KM, Mantzoros CS, Manson JE, Willett WC, et al. (2009) Mediterranean diet and incidence of and mortality from coronary heart disease and stroke in women. Circulation 119: 1093-1100.

110. Esposito K, Ciotola M, Giugliano F, De Sio M, Giugliano G, et al. (2006) Mediterranean diet improves erectile function in subjects with the metabolic syndrome. Int J Impot Res 18: 405-410.

111. Giugliano F, Maiorino MI, Bellastella G, Autorino R, De Sio M,et al. (2010) Adherence to mediterranean diet and erectile dysfunction in men with type 2 diabetes. J Sex Med 7: 1911-1917.
112. Inman BA, Sauver JL, Jacobson DJ, McGree ME, Nehra A, et al. (2009) A population-based, longitudinal study of erectile dysfunction and future coronary artery disease. Mayo Clin Proc 84: 108-113.

113. Nehra A, Jackson G, Miner M, Billups KL, Burnett AL, et al. (2012) The princeton iii consensus recommendations for the management of erectile dysfunction and cardiovascular disease. Mayo Clin Proc 87: 766-778.

114.Greenland P, Alpert JS, Beller GA, Benjamin EJ, Budoff MJ, et al. (2010) 2010 accf/aha guideline for assessment of cardiovascular risk in asymptomatic adults: Executive summary: A report of the american college of cardiology foundation/american heart association task force on practice guidelines. Circulation 122: 2748-2764

115. Araujo AB, Hall SA, Ganz P, Chiu GR, Rosen RC, et al. (2010) Does erectile dysfunction contribute to cardiovascular disease risk prediction beyond the framingham risk score? J Am Coll Cardiol 55: 350-356.

116. Gibbons RJ, Balady GJ, Bricker JT, Chaitman BR, Fletcher GF, et al. (2002) Acc/aha 2002 guideline update for exercise testing: Summary article. A report of the american college of cardiology/american heart association task force on practice guidelines (committee to update the 1997 exercise testing guidelines). J Am Coll Cardiol 40:1531-1540. 